\title{
Avaliação epidemiológica das fraturas da coluna torácica e lombar dos pacientes atendidos no Serviço de Ortopedia e Traumatologia do Hospital Getúlio Vargas em Recife/PE
}

\author{
Epidemiological evaluation of the thoracic and lumbar spine fractures \\ of patients treated in the Orthopedics and Traumatology Service at \\ Hospital Getúlio Vargas in Recife/PE \\ Evaluación epidemiológica de las fracturas de la columna torácica \\ y lumbar de los pacientes atendidos en el Servicio de Ortopedia y \\ Traumatología del Hospital Getúlio Vargas en Recife/PE
}

\author{
André Flávio Freire Pereira' \\ Luiz Eduardo Duque Portela ${ }^{2}$ \\ Guilherme Didier de Andrade Lima ${ }^{3}$ \\ Wagner Cabral Gomes Carneiro ${ }^{4}$ \\ Marcus André Costa Ferreira ${ }^{5}$ \\ Túlio Albuquerque de Moura Rangel ${ }^{6}$ \\ Ricardo Barreto Monteiro dos Santos ${ }^{7}$
}

\section{RESUMO}

Introdução: as fraturas da coluna torácica e lombar estão se tornando mais frequentes, devido ao aumento dos acidentes de alta energia. Elas apresentam um elevado índice de morbidade e mortalidade, acarretando grandes prejuízos socioeconômicos. Objetivo: analisar a epidemiologia das fraturas torácicas e lombares de pacientes atendidas no Serviço de Ortopedia e Traumatologia do Hospital Getúlio Vargas em Recife (PE). Métodos: este estudo é uma análise epidemiológica dos pacientes com fraturas da coluna torácica e lombar, admitidos na emergência do Hospital Getúlio Vargas no período de $1^{\circ}$ de novembro de 2007 a 30 de abril de 2008. Foram admitidos 42 pacientes com fra-

\section{ABSTRACT}

Background: thoracic and lumbar fractures have been more frequent, due to the increase of energy accidents. It causes a high index of morbidity and mortality and economical and social damage. Objective: to analyze the epidemiology of thoracic and lumbar fractures in patients attended at the Hospital Getúlio Vargas, in Recife (Pernambuco). Methods: this study is an epidemiological analysis of the thoracic and lumbar fractures in patients, who were admitted at the emergency room of Hospital Getúlio Vargas in the period between November $1^{\text {st }}$ of 2007 and April $30^{\text {th }}$ of 2008. Forty-two patients were admitted with thoracic and lumbar

\section{RESUMEN}

Introducción: las fracturas de la columna torácica y lumbar están más frecuentes debido al aumento de los accidentes de alta energía. Estas presentaron un índice elevado de morbilidady mortalidad, acarreando grandes daños socioeconómicos. Objetivo: analizar la epidemiología de las fracturas torácicas y lumbares de pacientes atendidas en el Servicio de Ortopedia y Traumatología del Hospital Getúlio Vargas, en Recife (PE). Métodos: este estudio es un análisis epidemiológico de los pacientes con fracturas de la columna torácica y lumbar, admitidos en la emergencia del Hospital Getúlio Vargas durante el periodo de 1 de noviembre de 2007 a 30 de abril de 2008. Fueron

\footnotetext{
Trabalho realizado no Serviço de Ortopedia e Traumatologia do Hospital Getúlio Vargas - Recife (PE), Brasil.

'Ortopedista e Traumatologista, Cirurgião de Coluna do Hospital Getúlio Vargas - Recife (PE), Brasil.

${ }^{2}$ Ortopedista e Traumatologista do Hospital Getúlio Vargas - Recife (PE), Brasil.

${ }^{3}$ Ortopedista e Traumatologista do Hospital Getúlio Vargas - Recife (PE), Brasil.

${ }^{4}$ Ortopedista e Traumatologista do Hospital Getúlio Vargas - Recife (PE), Brasil.

${ }^{5}$ Ortopedista e Traumatologista, Cirurgião de Coluna do Hospital Getúlio Vargas - Recife (PE), Brasil.

${ }^{6}$ Ortopedista e Traumatologista, Cirurgião de Coluna do Hospital Getúlio Vargas - Recife (PE), Brasil.

${ }^{7}$ Médico Residente de Ortopedia e Traumatologia do Hospital Getúlio Vargas - Recife (PE), Brasil.
} 
turas da coluna torácica e lombar, perfazendo um total de 54 vértebras fraturadas. Resultados: observou-se que as fraturas da coluna torácica e lombar foram mais frequentes no gênero masculino, com uma média de idade de 39 anos. As quedas de altura foram responsáveis por $61,9 \%$ dos casos, seguida dos acidentes com motos $(19,05 \%)$. Lesões neurológicas estiveram presentes em $14,28 \%$ dos casos e o tratamento cirúrgico foi realizado em $50 \%$ deles. Conclusão: as fraturas da coluna torácica e lombar, no presente estudo, foram frequentes nos adultos jovens, do gênero masculino, trabalhadores e causadas por queda de altura. fractures, they had a total of fiftyfour vertebral fractures. Results: thoracic and lumbar fractures were more often in men. The average age was 39 years old. The most frequently lesion mechanism was injuries from falls (61.9\%), followed by motorcycle accidents (19.05\%). Neurological injuries occurred in $14.28 \%$ of the cases and $50 \%$ of the patients needed surgery. Conclusion: thoracic and lumbar fractures, in the present study, were more frequent in men, young adults, workers and they were caused by high falls. admitidos 42 pacientes con fracturas de la columna torácica y lumbar, haciéndose un total de 54 vértebras fracturadas. Resultados: se observaron que las fracturas de la columna torácica y lumbar fueron más frecuentes en el género masculino, con una edad promedio de 39 años. Las caídas por altura fueron responsables por el 61,9\% de los casos, seguida por accidentes con motos (19,05\%). Lesiones neurológicas fueron en un 14,28\% y el tratamiento quirúrgico fue realizado en $50 \%$ de los casos. Conclusión: las fracturas de la columna torácica y lumbar, en el presente estudio, fueron frecuentes en los adultos jóvenes, del género masculino, trabajadores y fueron causadas por caída de una determinada altura.

\section{DESCRITORES: Coluna}

vertebral; Fraturas da coluna vertebral/epidemiologia; Vértebras torácicas/lesões; Vértebras lombares/lesões; Traumatismos da coluna vertebral/epidemiologia
KEYWORDS: Spine; Spinal

fractures/epidemiology; Thoracic vertebrae/injuries; Lumbar vertebrae/injuries; Spinal injuries/epidemiology
DESCRIPTORES: Columna vertebral; Fracturas de la columna vertebral/ epidemiología; Vértebras torácicas/lesiones; Vértebras lumbares/lesiones; Traumatismos vertebrales/ epidemiología

\section{INTRODUÇÃO}

Observa-se um aumento de pacientes vítimas de traumatismos na coluna vertebral, sendo elevado o índice de morbidade e mortalidade. A maioria dos pacientes são adultos jovens, trabalhadores, acarretando uma grande perda socioeconômica para a sociedade e para o Estado.

Estima-se que 15 a $20 \%$ dos pacientes com fratura nas colunas torácica e lombar apresentam lesão neurológica que exigem tratamento ${ }^{1}$. Nos Estados Unidos, ocorrem cerca de 11.000 novas lesões na medula espinhal por ano ${ }^{1}$. Estudos mostram que 40,1 habitantes dos EUA, em cada grupo de um milhão, sofram trauma da coluna torácica ou lombar a cada $a^{2}{ }^{2}$. No Canadá, a incidência é de 64 pacientes por 100.000 habitantes por ano ${ }^{3}$. Cunha, Menezes e Guimarães apresentaram uma incidência de 11,8 lesões traumáticas da coluna torácica e lombar por milhão de habitantes na região metropolitana de Belo Horizonte ${ }^{4}$.

Há grande importância no diagnóstico precoce dessas lesões, pois o déficit neurológico é sete vezes mais frequente em pacientes com diagnostico tardio ${ }^{1}$.

As causas mais frequentes das lesões da coluna vertebral são os acidentes de trânsito (45\%), seguido pelas quedas $(20 \%)$, pela prática de esportes $(15 \%)$ e por atos de violência $(15 \%)^{1}$.
No Brasil, são poucos os relatos epidemiológicos sobre fraturas da coluna toracolombar. No Brasil, na região Nordeste, não há trabalhos em publicações nacionais abordando o assunto. Este trabalho tem por objetivo realizar uma avaliação epidemiológica das fraturas das colunas torácica e lombar, em um hospital terciário na cidade do Recife.

\section{MÉTODOS}

Este estudo é uma análise epidemiológica de pacientes com fraturas da coluna torácica e lombar, atendidos na emergência do Hospital Getúlio Vargas no período de $1^{\circ}$ de novembro de 2007 a 30 de abril de 2008. Foram admitidos 42 pacientes com fraturas da coluna torácica e lombar, perfazendo um total de 54 vértebras fraturadas.

O formulário próprio da pesquisa foi preenchido durante o atendimento e/ou internamento dos pacientes, não sendo necessário recorrer ao Serviço de Arquivos Médicos e de Estatística (SAME) do hospital.

O formulário abordava aspectos epidemiológicos como idade, gênero, cor, município de residência do paciente, mecanismo do trauma, ingestão de bebida alcoólica no momento do trauma, presença e discriminação de lesões associadas extravertebrais, topografia e classificação das fraturas baseadas nos métodos de imagem disponíveis, 
presença e classificação de lesões neurológicas e métodos de tratamento empregados.

Todos os pacientes com suspeita de fraturas da coluna toracolombar foram submetidos a exames radiográficos, com imagem em anteroposterior e lateral. Quando foi observada alguma fratura, esses pacientes foram encaminhados para a realização de tomografia computadorizada para uma melhor avaliação da fratura. Em alguns casos, os pacientes ainda foram submetidos à ressonância magnética.

A classificação utilizada foi a de Margerl et al. ${ }^{5}$, adotada pelo Grupo AO, que agrupa as fraturas toracolombares em três grandes tipos: A (lesões por compressão do corpo vertebral), B (lesões por distração dos elementos anterior e/ou posterior) e C (lesões tipo A ou B com rotação e luxações complexas). Cada tipo, por sua vez, é subdividido em três subtipos: 1, 2 e 3. E cada subtipo é dividido em três subgrupos: 1, 2 e 3. Logo, essa classificação engloba um total de 27 tipos de fraturas. Essa classificação é a que apresenta a maior capacidade de englobar um maior número de fraturas. No presente estudo, optou-se por restringir a classificação aos tipos e subtipos, tendo em vista que esta classificação completa, com os seus subgrupos, é complexa, de difícil aplicação e apresenta elevado índice de discordância intra e interobservadores ${ }^{6-8}$. Então, as possíveis classificações incluíam: A1, A2, A3, B1, B2, B3, $\mathrm{C} 1, \mathrm{C} 2$ e C3. Não se aplicou esta classificação em fraturas por projétil de arma de fogo (PAF) da coluna toracolombar.

Os mecanismos de trauma foram divididos em seis grupos: acidentes automobilísticos, acidentes motociclísticos, queda de altura (não era especificado a altura), lesão por PAF, mergulho em águas rasas e outros mecanismos.

A avaliação neurológica foi realizada por meio da análise e graduação de todos os grupos musculares. A fraqueza muscular foi graduada de acordo com o método do Medical Research Council (MRC) ${ }^{9}$ (Tabela 1). A sensibilidade foi testada, incluindo tato, propriocepção e vibração. Todos os dermátomos foram avaliados bilateralmente e os reflexos, documentados.

\section{TABELA 1 - Graduação de força muscular}

\begin{tabular}{lc}
\hline Grau & Parâmetro testado \\
\hline 0 & Paralisia total \\
1 & Contração fracamente detectável \\
2 & Força insuficiente para atuar contra gravidade \\
3 & Força suficiente para atuar contra a gravidade \\
4 & Força presente, porém ainda não é normal \\
5 & Força normal \\
\hline
\end{tabular}

Fonte: Ruedi TP, Murphy WM, organizadores. Princípios AO do tratamento de fraturas. Porto Alegre: Artmed; 2002.

As lesões neurológicas foram agrupadas com base na classificação adotada pela American Spinal Injury Association (ASIA) e na International Medical Society of Paraplegia, que é um refinamento da classificação funcional original de Frankel: A (lesão completa), B (preservação sensitiva incompleta), $\mathrm{C}$ (preservação motora incompleta $\leq \mathrm{MRC}$ grau 3), D (preservação motora incompleta $>$ MRC grau 3) e E (função neurológica normal).
O método de tratamento foi dividido em dois grandes grupos: conservador ou cirúrgico. O método conservador inclui colete de Jewett ou observação. Já o método cirúrgico englobava parafusos pediculados e fixadores internos.

A indicação para o tratamento cirúrgico foi baseada nos critérios de instabilidade de White e Panjabi. Os pacientes que obtiveram um somatório maior ou igual a cinco pontos foram submetidos ao tratamento cirúrgico. $\mathrm{O}$ uso de fixadores internos foi indicado para os pacientes com lesão ligamentar posterior e/ou fraturas por explosão tipo A3.

Os pacientes foram agrupados quanto à cor em três grupos: brancos, pardos e negros. A classificação subjetiva foi realizada pelo grupo deste trabalho.

Foi questionado o município de residência dos pacientes, sendo então classificados em quatro grupos: Recife, Região metropolitana do Recife, Interior do Estado de Pernambuco e outros estados.

Os pacientes foram interrogados sobre a ingestão alcoólica no momento do trauma.

Os pacientes que apresentavam fraturas extravertebrais foram avaliados pela equipe de trauma do Hospital Getúlio Vargas, tendo o seu tratamento direcionado para o tipo específico de fratura.

Utilizaram-se, para armazenar as informações em banco de dados, os softwares Excel 2003 e Statistical Package for the Social Sciences - SPSS v. 11.0. Foram considerados estatisticamente significativos aqueles resultados, no teste do $\chi^{2}$, com o $\mathrm{p}<0,05$.

\section{RESULTADOS}

A idade média dos pacientes foi de 39 anos, sendo o desvio padrão de 15,1 anos, com idade mínima de 17 anos e máxima de 70 anos.

Quanto ao gênero, 31 eram do masculino (73,8\%), e 11 do feminino $(26,2 \%)(\mathrm{p}=0,002)$.

Com relação à cor, $35,71 \%(\mathrm{n}=15)$ eram brancos, $16,67 \%$ $(n=7)$ eram negros, e $47,62 \%(n=20)$ eram pardos $(p=0,046)$.

Observou-se que 45,24\% ( $\mathrm{n}=19)$ dos pacientes residiam em municípios que compõem a região metropolitana do Recife, 38,09\% ( $\mathrm{n}=16)$ residiam no Recife e $16,67 \%$ $(n=7)$ no interior do Estado de Pernambuco $(p=0,062)$ (Gráfico 1). Embora houvesse predomínio dos pacientes

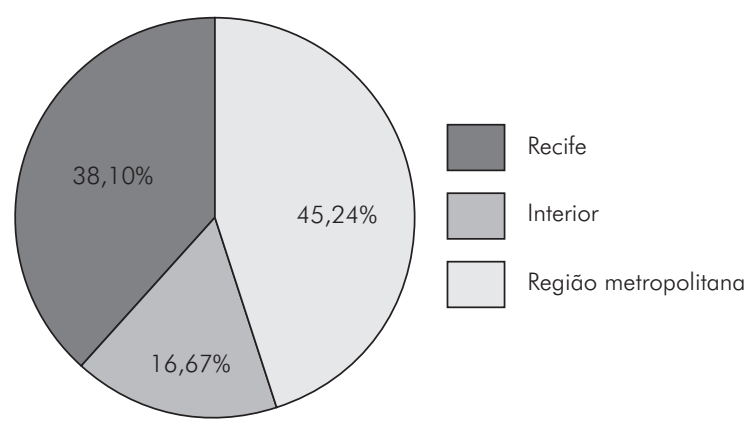

Gráfico 1

Distribuição geográfica dos pacientes que apresentaram fraturas da coluna torácica e lombar 
oriundos de municípios da região metropolitana do Recife, essa diferença não foi estatisticamente significante $(\mathrm{p}=0,062)$. Não foi observado nenhum paciente proveniente de outro Estado.

O mecanismo mais frequente foi acidente por queda de altura com $61,91 \%(\mathrm{n}=26)$, seguido por acidentes com motos $19,05 \%(\mathrm{n}=8)$, acidentes automobilísticos com 4,76\% $(\mathrm{n}=2)$, acidentes por PAF com $4,76 \%(\mathrm{n}=2)$ e outros mecanismos com 9,52\% $(n=4)(p<0,01)$ (Tabela 2).

\section{TABELA 2 - Distribuição de frequencia e} percentagem dos pacientes com fratura da coluna torácica e lombar quanto ao mecanismo de trauma

\begin{tabular}{lcc}
\hline Mecanismo & No de casos $^{\circ}$ & $\%$ \\
\hline Automobilísco & 2 & 4,76 \\
Motociclístico & 8 & 19,05 \\
Queda de altura & 26 & 61,91 \\
PAF & 2 & 4,76 \\
Outros & 4 & 9,52 \\
Total & 42 & 100 \\
\hline
\end{tabular}

Notou-se que $80,95 \%(n=34)$ dos pacientes referiram não ingerirem bebidas alcoólicas antes dos acidentes $(p<0,01)$. Dos oito $(19,05 \%)$ pacientes que referiram ingestão alcoólica antes do trauma, $50 \%$ apresentaram como mecanismo de trauma acidentes motociclísticos e 50\% queda de altura (Gráfico 2).

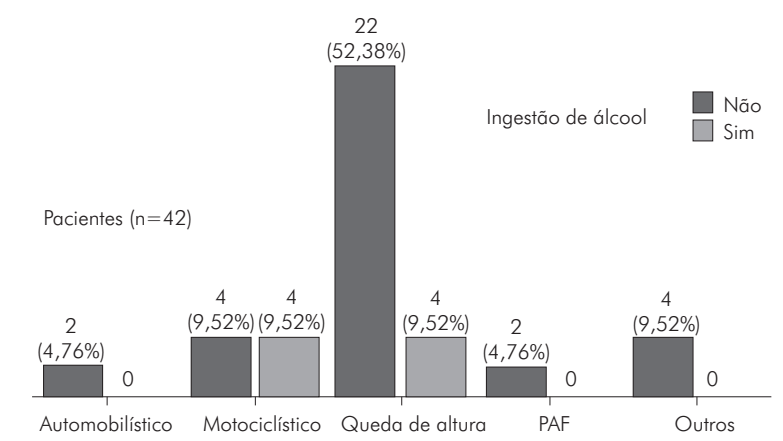

Gráfico 2

Distribuição dos pacientes com relação ao mecanismo de trauma e ingestão alcoólica

O estudo contabilizou 54 vértebras fraturadas nos 42 pacientes, sendo 34 lesões isoladas, oito lesões múltiplas (cinco lesões duplas, duas lesões em três vértebras, e uma em quatro vértebras). Dos cinco casos em lesões duplas, quatro foram em vértebras adjacentes e uma lesão foi nãocontígua. Dos dois pacientes com lesões em três vértebras, um apresentou lesões em vértebras adjacentes e o outro em vértebras não-contíguas. E o paciente com lesão em quatro vértebras apresentou lesões em vértebras não-contíguas.
A vértebra mais fraturada foi $\mathrm{L} 1 \mathrm{com} 38,9 \%(\mathrm{n}=21)$ das lesões, seguida pela vértebra T12 com 18,5\%, L2 com $11,1 \%(\mathrm{p}<0,01)$. Quando se realiza a comparação entre as proporções das vértebras torácicas fraturadas, observa-se predomínio da vértebra T12 com 55,6\% dos casos, seguida por $\mathrm{T} 11$ com $16,7 \%(\mathrm{p}=0,005)$. Realizando-se a mesma análise para o segmento lombar, há um predomínio de L1 com $58,3 \%$ dos casos, seguido por L2 com $16,7 \%(p<0,01)$ (Gráfico 3).

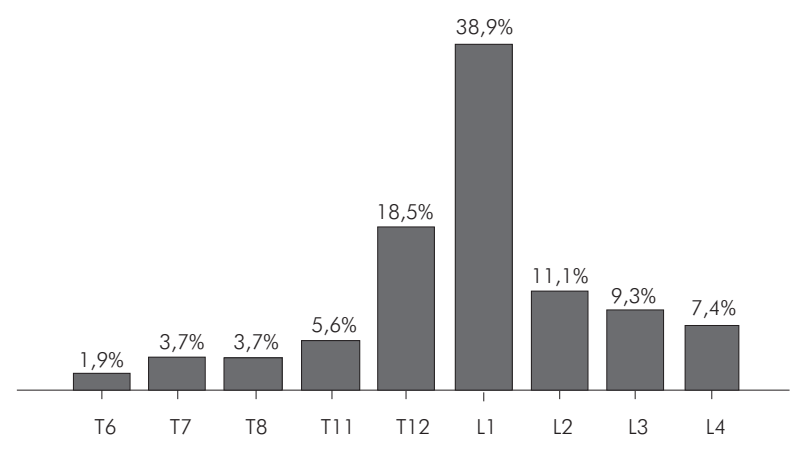

Gráfico 3

Análise percentual das vértebras fraturadas

Com relação ao segmento vertebral acometido, a coluna lombar foi acometida em $66,77 \%$ dos pacientes $(\mathrm{n}=36)$, seguida pela torácica em $33,3 \%(\mathrm{n}=18)$. A diferença entre as proporções foi estatisticamente significativa $(\mathrm{p}=0,014)$.

Quando se realizou a classificação das 54 vértebras fraturas, observou-se um predomínio do tipo A com $88,9 \%$ $(\mathrm{n}=48)$, seguido pelo tipo $\mathrm{C}$ com $5,6 \%(\mathrm{n}=3)$ e tipo B com $1,9 \%(\mathrm{n}=1)$. Houve diferença estatisticamente significativa $(\mathrm{p}<0,01)$. Dentre as fraturas do tipo A, houve um predomínio do subtipo A1 com 56,3\% ( $\mathrm{n}=27)$, seguido pelo subtipo A3 com 33,3\% $(n=16)$ e A2 com $10,4 \%(n=5)(p<0,001)$. As fraturas do tipo $\mathrm{C}$ foram todas do subtipo $\mathrm{C} 1$, e a do tipo B foi subtipo B1.

A lesão neurológica estava presente em $14,28 \%$ dos casos $(n=6)$, e $85,72 \%$ dos pacientes $(n=36)$ não apresentaram lesões neurológicas, sendo classificados como Frankel E. Essa diferença foi estatisticamente significativa $(\mathrm{p}<0,001)$. Dos seis pacientes com lesão neurológica, observou-se um paciente com Frankel A, dois com Frankel B, um com Frankel C e dois com Frankel D.

A evolução do quadro neurológico destes pacientes continua sendo acompanhada no ambulatório de Cirurgia da Coluna do Departamento de Ortopedia e Traumatologia do Hospital Getúlio Vargas em Recife (PE), não sendo relatada no presente estudo devido ao pouco tempo de seguimento e por não constar nos objetivos atuais. Estes dados, como demais condições dos pacientes, serão objetivos de trabalhos futuros.

As lesões extravertebrais foram observadas em nove $(21,43 \%)$ pacientes, totalizando 11 lesões. A lesão mais comum foi a fratura do calcâneo, com quatro $(36,3 \%)$ pacientes. As outras lesões foram duas no TGI, uma fratura 
no terço distal do rádio, um TCE, duas fraturas no ilíaco e uma fratura no tornozelo.

Com relação ao tratamento empregado, observou-se que $50 \%$ dos pacientes $(n=21)$ foram submetidos a tratamento cirúrgico e $50 \%(\mathrm{n}=21)$ foram tratados de maneira conservadora.

\section{DISCUSSÃO}

O presente estudo confirma dados que são apresentados na literatura ${ }^{3,10-12}$, em que os pacientes com fraturas da coluna torácica e lombar são, em sua maioria, adultos (idade média de 39 anos), economicamente ativos, do gênero masculino.

A população local é fruto de uma grande miscigenação de raças, sendo difícil enquadrar um paciente entre branco, pardo e negro. No estudo, observou-se um predomínio de pardos.

Os pacientes com lesões na coluna vertebral necessitam de um atendimento em hospital terciário, que seja capaz de dar o suporte adequado ao tratamento. Mais de $60 \%$ dos pacientes foram encaminhados de outros municípios, demonstrando que o Hospital Getúlio Vargas, localizado na cidade do Recife, é uma referência, no Estado de Pernambuco, no tratamento dessas lesões.

A queda de altura foi o mecanismo de trauma mais frequente encontrado, concordando com alguns trabalhos nacionais ${ }^{13,14}$ e internacionais ${ }^{3}$. Entretanto, outros autores afirmam que o mecanismo mais prevalente é o acidente automobilístico ${ }^{1,15}$, perfazendo até $70 \%$. Já nos pacientes idosos, é consenso na literatura que as quedas são responsáveis pela grande maioria das fraturas da coluna vertebral, havendo, inclusive, um maior acometimento do gênero feminino ${ }^{1,3,16}$.

A literatura demonstra uma incidência que varia de 15 a $40 \%$ de pacientes com fratura da coluna torácica e lombar com lesão neurológica associada ${ }^{1,8,14}$. Foi observado, no estudo, que $14,28 \%$ dos pacientes apresentavam lesões neurológicas associadas, variando desde Frankel D a Frankel A.

As lesões associadas estão presentes em 38\% dos pacientes $^{3}$. Até $25 \%$ dos pacientes com traumatismo na coluna têm, pelo menos, um TCE leve ${ }^{13}$. Em $20 \%$ dos pacientes com lesões na coluna lombar observam-se lesões nas extremidades ${ }^{3}$. Observaram-se, no estudo, lesões extravertebrais em $21,43 \%$ dos pacientes. A lesão mais comum foi a fratura de calcâneo, com 36,3\% das lesões associadas. As lesões ocorreram nas extremidades em $54,5 \%$ dos casos. Com exceção daquelas causadas por PAF, todas as demais lesões associadas foram causadas por queda de altura. As lesões do aparelho gastrintestinal foram observadas nos pacientes com lesões por PAF. O TCE foi notificado em apenas um paciente, porém, em alguns prontuários, não havia o registro da Escala de Coma de Glasgow na ficha de admissão do paciente no setor de emergência. Por isso, acredita-se que haja uma maior incidência de TCE associada a lesões na coluna vertebral.
É de consenso na literatura que as vértebras mais acometidas estão localizadas na transição toracolombar, ${ }^{3,13,16}$. A grande maioria das fraturas por compressão ocorre entre as vértebras $\mathrm{T} 11$ e L2 ${ }^{17}$. A coluna toracolombar possui três regiões anatômicas e biomecânicas distintas: coluna torácica alta (T1-T10), junção toracolombar (T11-L2) e coluna lombar (L3-L5). Cada uma deve ser considerada separadamente, pois cada região apresenta diferentes taxas de ocorrência e padrões de fraturas, lesões neurológicas e métodos de tratamento. A junção toracolombar é particularmente suscetível a lesões. Fraturas nesse nível representam 50\% de todas as lesões na coluna, excetuando-se as ocorridas na coluna cervical. A transição de um segmento firme (coluna torácica alta) para um móvel (coluna lombar) cria um elevado estresse na junção toracolombar. A mudança da cifose torácica para lordose lombar deixa a transição toracolombar suscetível a cargas axiais ${ }^{18}$. Esse fato foi comprovado no estudo, pois a vértebra mais fraturada foi L1 (38,9\%), seguida pela vértebra T12 e L2. A junção toracolombar, que compreende de T11 a L2, foi o segmento acometido em $74 \%$ das fraturas da coluna toracolombar. Estima-se que fraturas vertebrais em vários níveis, que podem ser contíguas ou separadas, ocorrem em 3 a 5\% dos pacientes com fratura da coluna vertebral ${ }^{16}$. Outras fontes afirmam que a incidência de uma segunda lesão vertebral é de até $20 \%$; assim, se uma lesão vertebral significativa foi identificada em um nível, o resto da coluna deve ser reexaminado clinicamente, e um exame radiográfico em incidência anteroposterior e laterais de toda a coluna vertebral devem ser obtidas9. Observou-se, no estudo, uma incidência de $19,04 \%$ de fraturas vertebrais em mais de um nível.

Com relação à classificação $\mathrm{AO}$, no trabalho original de Aebi et al. ${ }^{19}$, as fraturas do tipo A perfazem um total de $66,16 \%$, sendo as $\mathrm{A} 1$ as mais frequentes $(52,51 \%)$, seguidas pelas A3 (42,26\%). As fraturas do tipo B representam $14,4 \%$ dos casos, e as do tipo C, $19,38 \%$. O presente estudo confirma o predomínio das fraturas do tipo A, porém com um percentual mais elevado $(88,9 \%)$. As fraturas do tipo A1 $(56,3 \%)$ foram confirmadas como as mais frequentes do Grupo A, seguidas pelas do tipo A3 (33,3\%). O segundo grupo mais observado foi o tipo $\mathrm{C}$ (5,6\% dos casos), sendo o Grupo B responsável por apenas $1,9 \%$ dos casos.

Foi observado que $19,05 \%$ (oito) dos pacientes referiam ingestão alcoólica antes do acidente. Esses pacientes apresentaram fraturas mais graves, pois $75 \%$ destes pacientes necessitaram tratamento cirúrgico. Notou-se que $50 \%(\mathrm{n}=4)$ dos pacientes que sofreram acidentes motociclísticos referiram ingestão alcoólica antes do trauma.

Os métodos clássicos de tratamento conservador para as fraturas da coluna torácica e lombar são: repouso no leito, redução incruenta por tração seguida de imobilização gessada e uso de órteses (colete de Jewett). Critérios de estabilidade com ausência de lesão neurológica, compressão do canal medular inferior a $50 \%$, cifose inferior a $30^{\circ}$ e ausência de translação vertebral são os critérios mais comumente utilizados para indicar o tratamento 
conservador. Deve-se, ainda, empregar esse tratamento naqueles pacientes que não apresentam condições clínicas para o tratamento cirúrgico ${ }^{1,13}$. O tratamento conservador foi empregado em $50 \%$ dos pacientes, obedecendo aos critérios descritos anteriormente. $\mathrm{O}$ método mais empregado foi o colete de Jewett, com quase a totalidade dos casos. Não foi utilizado nenhum aparelho gessado, tendo em vista o maior conforto do paciente, já que a cidade do Recife apresenta altas temperaturas durante quase todo o ano.

O tratamento cirúrgico foi empregado nos outros $50 \%$ dos pacientes. A indicação foi baseada nos critérios de instabilidade de White e Panjabi; os pacientes que obtiveram um somatório maior ou igual a cinco pontos foram submetidos ao tratamento cirúrgico. Os métodos empregados foram os parafusos pediculados e o fixador interno.

\section{CONCLUSÕES}

As fraturas da coluna torácica e lombar, no presente estudo, ocorreram mais comumente nos adultos jovens, do sexo masculino, trabalhadores e foram causadas por queda de altura.

A ingestão de bebida alcoólica esteve presente em apenas $19,05 \%$ dos casos, porém associou-se com fraturas mais graves, inclusive com o aumento da necessidade de tratamento cirúrgico.

\section{REFERÊNCIAS}

1. Bucholz RW, Heckman JD. Rockwood e Green: fraturas em adultos. 5a ed. São Paulo: Manole; 2006. p. 1405-58.

2. Bracken MB, Freeman DH Jr, Hellenbrand $\mathrm{K}$. Incidence of acute traumatic hospitalized spinal cord injury in the United States, 1970-1977. Am J Epidemiol. 1981;113(6):615-22.

3. Hu R, Mustard CA, Burns C. Epidemiology of incident spinal fracture in a complete population. Spine (Phila Pa 1976). 1996;21(4):492-9.

4. Cunha FM, Menezes CM, Guimarães EP. Lesões traumáticas da coluna torácica e lombar. Rev Bras Ortop. 2000;35(1/2):17-22.

5. Magerl F, Aebi M, Gertzbein SD, Harms J, Nazarian S. A comprehensive classification of thoracic and lumbar injuries. Eur Spine J. 1994;3(4):184201.

6. Wood KB, Khanna G, Vaccaro AR, Arnold PM, Harris MB, Mehbod AA. Assessment of two thoracolumbar fracture classification systems as used by multiple surgeons. J Bone Joint Surg Am. 2005;87(7):1423-9.

7. Oner FC, Ramos LM, Simmermacher RK, Kingma PT, Diekerhof CH, Dhert WJ, et al. Classification of thoracic and lumbar spine fractures: problems of reproducibility. A study of 53 patients using CT and MRI. Eur Spine J. 2002;11(3):235-45.
8. Kriek JJ, Govender S. AOclassification of thoracic and lumbar fractures - reproducibility utilizing radiographs and clinical information. Eur Spine J. 2006;15(8):1239-46.

9. Ruedi TP, Murphy WM, organizadores. Principios AO do tratamento de fraturas. Porto Alegre: Artmed Editora; 2002. p. 601-15.

10.Puertas EB, Chagas JCM, Mercurio R, Milani A. Fraturas da coluna vertebral na região toracolombar: estudo de 36 casos. Rev Bras Ortop. 1991;26(6):196-200.

11. Solino JL, Melo MFFV, Silva DHA, Elias N. Traumatismos da coluna vertebral. Avaliação da etiologia, incidência e frequência. Rev Bras Ortop. 1990;25(3):185-90.

12.Oliveira PAS, Pires JV, Borges Filho JMM. Traumatismos da coluna torácica e lombar. Avaliação epidemiológica. Rev Bras Ortop. 1996;31(9):771-6.

13.Zaninelli EM, Graells XSI, Néri OJ, Dau L. Avaliação epidemiológica das fraturas da coluna torácica e lombar de pacientes atendidos no Pronto-Socorro do Hospital do Trabalhador da UFPR de Curitiba - Paraná. Coluna/Columna. 2005;4(1):11-5.

14.Koch A, Graells XSI, Zaninelli EM. Epidemiologia de fraturas da coluna de acordo com o mecanismo de trauma: análise de 502 casos. Coluna/ Columna. 2007;6(1):18-23.
15.Pierce DS, Nickel VH, editors. The total care of spinal cord injuries. Boston: Little, Brown; 1977.

16. Canale ST, editor. Cirurgia ortopédica de Campbell. 10a ed. Barueri: Manole; 2007. vol. 2. p. 1642-75.

17. Vaccaro AR, Kim DH, Brodke DS, Harris M, Chapman J, Schildhauer T, et al. Diagnosis and management of thoracolumbar spine fractures. Instr Course Lect. 2004;53:359-73.

18.Mikles MR, Stchur RP, Graziano GP. Posterior instrumentation for thoracolumbar fractures. J Am Acad Orthop Surg. 2004;12(6):424-35. Review.

19.Aebi M, Thalgott JS, Webb JK, editors. AO ASIF principles in spine surgery. Berlin: Springer; 1998.

Correspondência
Luiz Eduardo Duque Portela
Rua José Carvalheira, 203/1.501 -
Tamarineira
CEP: 52051-060 - Recife (PE), Brasil
Tel.: (81) 3268-0620 / (81) 9480-1326
E-mail: luiz_portela@hotmail.com

